\title{
Congestion Control for Better Performance of WSN Based IoT Ecosystem using KHA Mechanism
}

\author{
Shiv. H. Sutar, Y.Bevish Jinila
}

\begin{abstract}
Since last two decades, Wireless Sensor Network and Mobile Communication success rate is raised too high and demanding for overall communication network as it consists of heterogeneous wireless networks. These networks provides universal wireless access change, also provide dynamic scheduling between devices, networks and whole world for communication. It is applicable for prominently highly demanding domains like medical devices, sensors used for military applications, smart buildings, societies, and cities so on. At the same time new technological domain named IoT (Internet of Things) is making high impact on communication domain. It is an integration of several technologies. It is interchangeably can also be used as Wireless Sensor Network only the difference is that the IoT devices and networks has limited storage and computing capabilities and works on the internet. As IoT Ecosystem and WSN used for numerous Computation, Communication over the internet which ultimately increased network traffic and leads to congestion in the network which affects the Quality of Service (QoS) parameters of the network. In this paper, a novel method is proposed to handle the increased network traffic and Congestion network using the Krill Herd Aggregation Mechanism.
\end{abstract}

Keywords - Wireless Sensor Network, IoT, QoS, Congestion, Krill Herd Aggregation, Fitness Function.

\section{INTRODUCTION}

Firstly there is less concern about the traffic and control over the traffic in the network as the WSN and IoT is in demand now a days. Secondly, many mechanisms suggested to handle the application traffic and to control the low power and in expensive sensor nodes. Thirdly, as the WSN and IoT applications can be categorized into different types based on types of data generation mechanism. The WSN network is made up of small sensors which have its data generation and computing capabilities based on their functionalities, the sensor nodes sense the data based on the type of data it processes the data and then forward it the intended destination node called Sink node[14,15,16]. As the data can be of Event Driven Data, Bursty Data, and Periodic Data. Ultimately these types of data will produce the packets of variable sizes and the processing node has to handle these types of data dynamically based on current traffic load and processing load on the nodes. The node might be sender node; in that case the node will produce the packets and send to the intermediate node for further actions. If it is an intermediate node then it will process data packet and send

Revised Manuscript Received on July 10, 2019.

Shiv. H. Sutar, Research Scholar, Sathyabama Institute of Science and Technology, Chennai, T.N, India.

Y.Bevish Jinila, Associate Professor, School of Computing, Sathyabama Institute of Science and Technology, Chennai, T.N, India. it over the network based on routing mechanism used by the network.

\section{LITERATURE SURVEY}

The aim of IoT is now to create a flawless network of billions of wireless devices that can communicate over the internet.IoT ecosystem has millions of components with enormous diversity ranges from small sensors to large, powerful data center nodes. This dynamic nature and unambiguous data generation (the data may be event driven, periodic data, busrty data) by any small or large smart objects, sensor nodes makes IoT Ecosystem very challenging.

IoT ecosystem has to acquire this data, transform the data and process the data to valuable information and ultimately it use all devices and communication network. Such processing needs resources from ecosystem based on their properties. Like some applications [3-6] may need high processing computing, some might need complicated data processing and some might be latency sensitive so on. But the major limitations of IoT components are the limited computing and energy resources and also not capable to carry out complicated tasks. So there is need of powerful components that carry out these transformation processes which is required by IoT applications. Such components may be smart devices, data centers so on. This leads to the requirement of Resource management which includes Performance Monitoring and Configuration of Networks.

The resource management interfaces are always placed at the front of IoT network. That provides all relevant information to the end user. As IoT era aims to connect smart objects components via Internet. The interconnection of objects and the data produced needs much more coordination communication, management, tracking of events, and Control. Also Wireless Sensor Network can play important role by collecting surrounding context and environmental Information. The main challenge of IoT [710] is the limitation of resources, Energy Supply, Processing Power Storage and Memory, Wireless Communication Range which ultimately affects the Routing of this packets and information, based on any routing mechanism used it may use AODV, DSDV AOMDV and DSR Routing Mechanism. Deploying this WSN configured IoT enabled Ecosystem raises many novel challenges. This ultimately

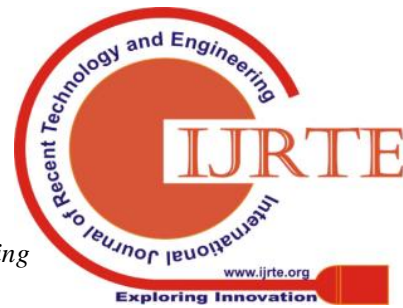




\section{CONGESTION CONTROL FOR BETTER PERFORMANCE OF WSN BASED IOT ECOSYSTEM USING KHA MECHANISM}

motivates us to discuss about one of the major challenges called contention or congestion in the network. This congestion in the network affects many Quality of Services Parameters of the Network that is IoT enabled WSN QoS Parameters.Many techniques have been proposed to handle congestion in the network - such as avoidance and mitigation techniques.Basically root causes of Congestion in the network found are - Input output flow rate, Node density, unbalanced distribution of load on the nodes, Processing time of each node, and so on. This also includes, if the data transmission rate of earlier - previous node is high than the processing speed of the node. So the nodes are basically provided with the Buffer which is used to store the packets before and after processing the packets until the node is capable to accept and forward it to the intermediate node. The buffers holds the packets but if still the incoming flow rate is more than the processing speed of the node the buffer occupancy will get full and the next packet arrived will be dropped from the buffer, this will lose the information carried by the packet. is congested so the output buffer cannot send the processed packets through the network and ultimately the processed packet which is to be stored will again is lost. So for buffer management different types of buffers are used in the wireless sensor network. Following types of buffers are Drop Tail Buffer, Fair Queuing Buffer, Stochastic Fair Queuing, Deficit Round Robin, Class Based Queuing, and So on. Each with its own properties plays important role in packet storing and forwarding in the communication network. Many authors have proposed and implemented various techniques for handling congestion in the network. Some of them are just mentioned for the better understanding of congestion avoidance and mitigation. Author for Local Cross Layer Congestion Control mechanism used for Congestion identification for based on Buffer Occupancy in which the Source Node acts as an action identifier who is responsible to identify the current occupancy of buffer and apply rate control that is it lower downs the packet generation rate so that buffer occupancy can be managed and buffer creates space for incoming packets.

ADCC is another mechanism suggested which uses a Congestion Degree for identification of Congestion and this identification is managed by intermediate nodes and uses duty cycle adjustment mechanism [11-12]. Another RACC mechanism here uses Round Trip Time Timer for identifying congestion in the network, where Sink Node used to detect the congestion and it is controlled by adjustment in the congestion window. Buffer Based Congestion Avoidance mechanism allows to detect the congestion in the network by using buffer overflow status, here source node takes an action and uses forwarding rate control mechanism for handling the congestion. ESRT is another mechanism used where it uses congestion notification bit to manage and handle congestion by managing Sink Node. [13] EDCAM- Early Detection Congestion Avoidance Mechanism used again buffer management, considering buffer as a queue where queue will be used with some threshold levels (High Water Mark and Low Water Mark Level) and based on the current status
And same if the processing speed is fast and the network

of the queue. The HWM and LWM notifies to the nodes when to lower down the flow rate and when to increase the flow rate of Packets as per the status of Buffer used as a queue. It also uses notification bit to adjust the flow rate of Packets. Again this congestion may be Node Level Congestion, in case of node level congestion the intermediate node may get over loaded and the buffer occupancy get full and next incoming packet may get dropped and ultimately this lost packet cannot be recovered. Another is Link Level Congestion in which multiple nodes sending the packets towards another node or the Sink node in which all the links get flooded with many packets leads to congestion. So we need to handle congestion in any means to control the Quality of Services of the network. In every case normal packet processing is previous node sends packet to next node, if next node is in position to accept the packet, it will occupy it in incoming buffer and then when complete its current packet processing the next packet in the queue will be taken for processing and forward it on the selected route by the routing protocol. And finally the packet will be received by the Sink Node.

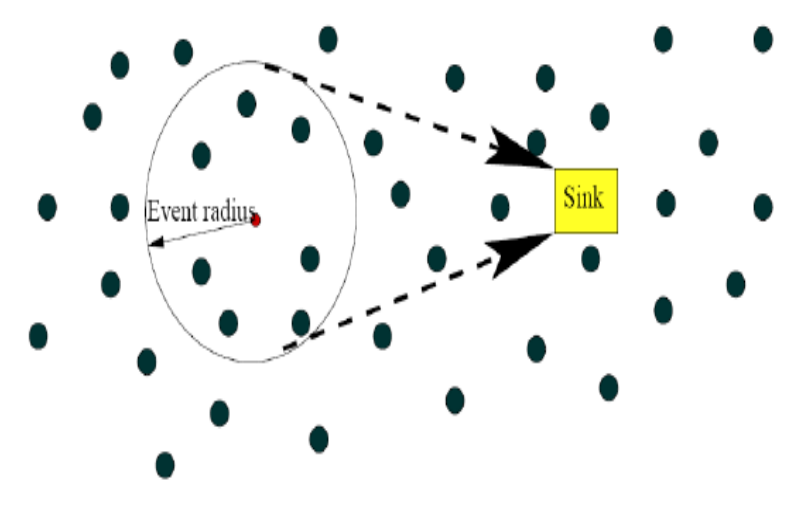

Fig.1 Node Distribution

Ultimately we need to think of optimization in the network to avoid congestion in the network eventually it will improve the QoS parameters of the network. [1] Shah and other have proposed an energy and interoperable aware routing for throughput optimization, decreasing the packet broadcast, lessoning computation at the node level.

[2] M. Shelke and others proposed a Packet Priority Based (PPI) congestion control mechanism in which introduced a PPI Bit in each packet by modifying the existing packet format and deployed in the AODV routing Protocol to convert the existing routing protocol to a congestion aware routing protocol.

\section{PROPOSED SCHEME \& RESULTS}

As WSN have progressively utilized in the IoT ecosystem for data collection processing and transformation in current era. Congestion is a complicated issue and it happens in all kinds of networks irrespective of how these networks are established because it consists of multiple small, large, smart devices, and it results in numerous data generation, 
processing and transformation to be done for getting and communicating proper information to the end user. Ultimately it generates huge traffic in the network which significantly reduces network performance and effects on other QoS Parameters like Throughput of the network, Endto-end Delay, Packet Delivery Ratio, Latency, Energy Consumption, Reliability, Packet Drop Ratio, Packet Loss Ratio, and Fairness too.

To overcome this issue of Congestion in the network, a Performance Optimization by Congestion Control for WSN based IoT Ecosystem using KHA Mechanism is proposed. This proposed mechanism is based on Krill Herd Aggregation algorithm which actually is based on simulation of the herding behavior of krill swarm individuals. The minimum distances of each individual krill from food and from the highest density of the herd are considered as the objective function for the krill movement. As the time dependent position of the Krill individuals is formulated by 3 main factors. First, Movement of Individuals by the presence of other individuals.

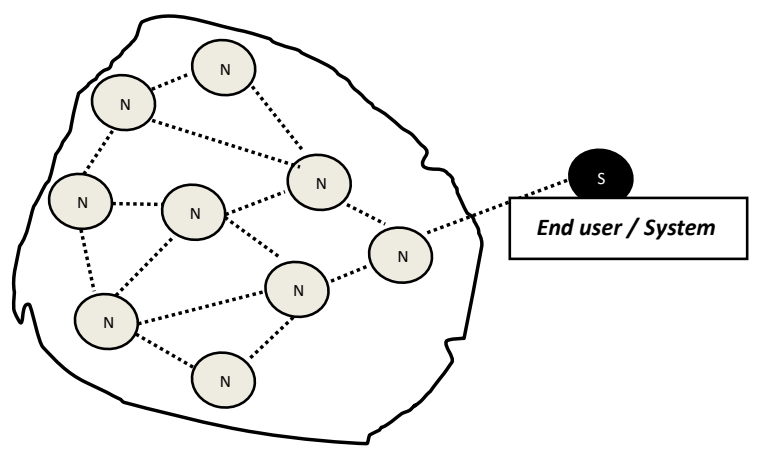

Fig.2. Proposed Scheme-KHA

Here the individuals will decide the movement due to induction that is other individuals movement, each krill to move towards optimal density as the density position will decide the possibility of food that swarm is looking for. Actually it uses sensing distance for sensing the neighbours. Second, Foraging Activity is decided by the previous distance for food and density and the current distance. Third, Random Diffusion is expressed as function phenomenon based on speed and directional approach for individual krill. Here idea is to take up this challenge of Krill Swarm to find food for each individuals based on these three phenomenon, we want to propose that the congestion can also be controlled by using the same terminology for the IoT enabled Wireless Sensor Network. As each node in the network is trying for generating the packets as data is sensed by the node, this data may be of any type, it needs to be acquired, processed and transformed by each node based on their processing computing and transforming capabilities. Here as each node is trying to send the sensed information to ultimate destination that is BASE STATION sometimes called Sink Node too. Here proposed system calculates the Fitness Function using QoS parameters to find out which node is capable of handling task of cluster head.

Parameters affecting the selection are No. of Nodes, Traffic, Euclidean Distance, and Energy of each node and calculates the Fitness Function.
Nx1- No. of Nodes, Tx2- Traffic, EDx3- Euclidean Distance, Px4-Energy.

By adding the constant values of these parameters the fitness function for each node is calculated. Simply a denoting nodes, $b$ - denoting traffic (frequency of packets), c- ED (Euclidean Distance) and d - denoting Energy. Will calculate Fitness Function (y), where,

$$
Y=a \times 1+b \times 2+c \times 3+d x 4
$$

For each node the Fitness Function will be calculated (like $\mathrm{n} 1=72 \%, \mathrm{n} 2=56 \%, \ldots . \mathrm{n} 6=84 \% \ldots .$. ).

The node having Maximum fitness function will be choosen as a cluster head. So these three main objective functions can be considered for such type of network. First, movement induced by the presence of individuals, here packet or data to be send to intermediate node will take the route in consultation with the routing protocol, as all routing protocols are greedy in nature they will try to choose shortest path from the source to the destination node (here Cluster Head) which is responsible to receive the packets from each source node and forward it to the desired sink Node, and the fitness function is calculated for the selection of cluster head using QoS parameters, whichever node is having highest value will be chosen as a leader node.

Ultimately they will choose the node will be more densed in terms of Food with Most affordable services, All Quality of Services parameters that is this node can also be called as a Cluster Head among the nodes. This cluster head will receive more and more packets which is capable to collect the packets and gather the information to be communicated to the Sink Node may be through other cluster node. Likewise the cluster head will be choosen optimally based on the how the packets are moving in the network through which link and to which node.

Again depending on previous data communicating channel or link used where cluster head can be directly found for further communication based on previous knowledge, unless new cluster head is choosen. The new cluster head will be choosen only when existing cluster head is not in position to accommodate and handle the traffic, which ultimately leads to congestion in the network. But as our algorithm says individuals will move to high density node which is the node with maximum available food of Energy, Processing Capabilities, Transmission range and other resources. Also the Speed of Data Transmission based on Transmission range that is Sensing Distance of each node for the neighbor node. So ultimately it will automatically avoid the congestion in the network. Which altogether reduces unnecessary traffic load in the network and the possible congestion in the network can be avoided. This proposed schema can be simulated using NS2 - Network Simulator 2. It is a discrete event simulator, it allows setting up an environment for the topology and allows configuring the network as per the requirement to check the performance of the network and generate the trace file which is helpful to analyze the overall performance of the network by changing different parameters. 


\section{CONGESTION CONTROL FOR BETTER PERFORMANCE OF WSN BASED IOT ECOSYSTEM USING KHA MECHANISM}

The simulation result will produce expected Quality of Services Parameters which will improve these QoS parameters by handling the congestion in the network. Ultimately proposed system will improve overall performance of the network by properly choosing the cluster head based on runtime capability of the node. At last aim is to increase the QoS parameters of the network $10 \%$ - 20\%.( Increase- PDR, Fairness, Throughput and also reducesPLR, E to E Delay, Energy Consumption) with help of WSN and IoT Ecosystem.

\section{CONCLUSION}

By controlling the congestion in the network using the proposed scheme, we can identify the cluster head - the node capable to handle the traffic load and communicate the information to the base station. We simulate this scheme using NS-2 simulator and the expected results can be compared with the simulated results to analyze the QoS parameters of the network for better performance. Later in future we wish to apply this proposed scheme along with other mechanism for better Performance, because of the proposed scheme some of the parameters may get hampered so we might need to rectify those parameters.

\section{REFERENCES}

1. S. Shah, Z. Chen, F. Yin, I. Khan and N. Ahmad, "Energy and interoperable aware routing for throughput optimization in clustered IoT-wireless sensor networks", Future Generation Computer Systems, vol. 81, pp. 372-381, 2018.

2. M. Shelke, A. Malhotra and P. Mahalle, "A packet priority intimation-based data transmission for congestion free traffic management in wireless sensor networks", Computers \& Electrical Engineering, vol. 64, pp. 248-261, 2017.

3. F. Li, Y. Han and C. Jin, "Practical access control for sensor networks in the context of the Internet of Things", Computer Communications, vol. 89-90, pp. 154-164, 2016.

4. Performance Analysis of Wireless Sensor Networks for QoS, International Conference on Big Data, IoT and Data Science (BID) Vishwakarma Institute of Technology, Dec 2017.

5. Chetana Pawar, Shiv Sutar, Snehal Umare, Minal Chaudhary "Increasing Network Lifetime using PDR Analysis and Fuzzy Logic in WSN" International Journal of Computer Engineering and Applications, Volume XII, Special Issue, March 2018, www.ijcea.com ISSN 2321-3469.

6. C. Tsai, "SEIRA: An effective algorithm for IoT resource allocation problem", Computer Communications, 2017.

7. P. Porambage, A. Braeken, C. Schmitt, A. Gurtov, M. Ylianttila and B. Stiller, "Group Key Establishment for Enabling Secure Multicast Communication in Wireless Sensor Networks Deployed for IoT Applications", IEEE Access, vol. 3, pp. 1503-1511, 2015.

8. J. Duan, D. Gao, D. Yang, C. Foh and H. Chen, "An EnergyAware Trust Derivation Scheme With Game Theoretic Approach in Wireless Sensor Networks for IoT Applications", IEEE Internet of Things Journal, vol. 1, no. 1, pp. 58-69, 2014.

9. R. Fantacci, T. Pecorella, R. Viti and C. Carlini, "A network architecture solution for efficient IOT WSN backhauling: challenges and opportunities", IEEE Wireless Communications, vol. 21, no. 4, pp. 113-119, 2014.

10. J. Huang, Y. Meng, X. Gong, Y. Liu and Q. Duan, "A Novel Deployment Scheme for Green Internet of Things", IEEE Internet of Things Journal, vol. 1, no. 2, pp. 196-205, 2014.

11. F. Li and P. Xiong, "Practical Secure Communication for Integrating Wireless Sensor Networks Into the Internet of
Things", IEEE Sensors Journal, vol. 13, no. 10, pp. 36773684, 2013.

12. Sandeep Saurav Singh,Y.Bevish Jinila," Sensor Node Failure Detection using Check Point Recovery Algorithm", Fifth International conference on recent trends in Information Technology (ICRTIT), 8-9th April 2016, IEEE.

13. Dr Helonde J. B. Dr. Wadhai V. Vivek Deshpande, Shiv Sutar Pune, India. "EDCAM: Early Detection Congestion Avoidance Mechanism for Wireless Sensor Network", International Journal of Computer Applications (0975 - 8887) Volume 7- No.2, September 2010.

14. Sivasangari, A., Bhowal, S., Subhashini, R., Secure encryption in wireless body sensor networks, Advances in Intelligent Systems and Computing, 2019.

15. Sethuraman, R., Hema, P., Gnana Priya, R. "Semantic web services based implementation of home appliances into ZigBee coordinators network" 2016 2nd International Conference on Science Technology Engineering and Management, ICONSTEM 2016.

16. Nandini, D.U., Saravanan, M., Mayan, J.A., Kamalesh, M.D., Prasad, K.M., Automatic traffic control system using PCA based approach, International Conference on Energy, Communication, Data Analytics and Soft Computing, ICECDS, 2018 